\title{
Effects of Waveform Model on Sensitivity Values of Transducers Used in Mechanical Dynamic Measurements
}

\author{
E. BilgiÇ ${ }^{a, *}$ AND Y. DURGUT ${ }^{b}$ \\ ${ }^{a}$ TÜBİTAK Ulusal Metroloji Enstitüsü, Acoustics Laboratory, Gebze-Kocaeli-Turkey \\ ${ }^{b}$ TÜBİTAK Ulusal Metroloji Enstitüsü, Pressure Laboratory, Gebze-Kocaeli-Turkey
}

\begin{abstract}
Dynamic calibration of the pressure transducers and accelerometers are carried out by applying dynamic mechanical inputs to them. Determination of those transducers' sensitivities, defined as the ratio of electrical output of the transducer to the mechanical input, is an important task for calibration laboratories. Data obtained during calibration are processed in order to have the peak values of the input and output signals which are sampled by data acquisition boards. Different approximations are made such as fitting the data in the range of $90 \%$ of the maximum value for parabola or half-sine waveform. It is clear that waveform model used and also the resolution and the sampling rate of the data acquisition boards have effects on the accuracy of the sensitivity of the transducer. For the investigation, the electrical output signal of the transducer corresponding to the mechanical input is recorded and simulated with different resolutions and sampling rates. Those data are processed for the waveforms of half-sine, parabola, Gaussian distribution. The effect of the waveform model of the input quantities on the dynamic sensitivity is discussed in this paper.
\end{abstract}

DOI: 10.12693/APhysPolA.128.B-267

PACS: 06.20.dk

\section{Introduction}

Many applications of the measurement of the mechanical quantities force, torque, and pressure are of a dynamic type, i.e. the measurement quantity shows a strong variation over time. In measurement systems; transducer, its conditioning amplifier and some other auxiliary equipment constitute measurement chain. Traceability of the dynamic measurements to SI units is established through the calibration of the pressure sensors. Metrological traceability is property of a measurement result whereby the result can be related to a reference through a documented unbroken chain of calibrations, each contributing to the measurement uncertainty [1]. The calibration of the respective transducers, however, is still only traceable to purely static procedures at the National Metrology Institute "NMI" level. This is partly due to the complete lack of documentary standards or commonly accepted guidelines for dynamic calibration of mechanical sensors, which is a consequence of the lack of a joint international research effort in the field [2]. In recent years, some of NMIs have made effort in order to make traceable dynamic measurements. Therefore, in order to cooperate between NMIs, a project in scope of European Metrology Research Program, EMRP "IND09 Dynamic: Traceable dynamic measurement of mechanical quantities" is going on. In scope of this paper dynamic pressure measurements performed using impact test machine which works based on drop mass principle. In order to determine the maximum peak value of the output of the measurement chain, several approximations made.

*corresponding author; e-mail: eyup.bilgic@tubitak.gov.tr

\section{Dynamic pressure and application fields}

There are some routine measurements in wide application area that such measurements show time varying characteristics because of their nature. Sudden pressure increase and decrease occurs inside the gun by means of firing the gun. Variation of pressure inside the gun by time is an example of dynamic pressure phenomena. Some other examples are pressure variation in car engines' cylinders, blood pressure in vessels, and crash tests, etc.

Increase in the demand for space and aeronautics fields after 1960s, research focused on advanced weapon systems, shock waves, measurements on explosion, rocket firing systems and ballistic tests have put forward the necessity for dynamic pressure sensors. Ballistic works, bio mechanical measurements, engine tests, engineering and industrial applications are the main areas where dynamic pressure measurement are used commonly. Measurement of time varying parameters is become quite necessary in many control processes. Amplitude and frequency interval are variables in dynamic measurements. In blast test, pressure and frequency parameters can reach up to $1 \mathrm{GPa}$ and $100 \mathrm{MHz}$, while working with $13 \mathrm{~Pa}$ and $50 \mathrm{kHz}$ values in space related issues [3].

Pressure transducer is an electromechanical convertor that converts mechanical input quantity "pressure" to electrical output quantity "charge" and consequence voltage. The conversion factor, named sensitivity, for a good quality linear transducer is constant in measurement range.

Primary and secondary level calibration methods for vibration and shock transducers are defined in ISO 16063 series standards. Especially for shock inputs, ISO 16063-13:2001 and ISO 16063-22:2005 standards are used. Method for determination of maximum peak value 


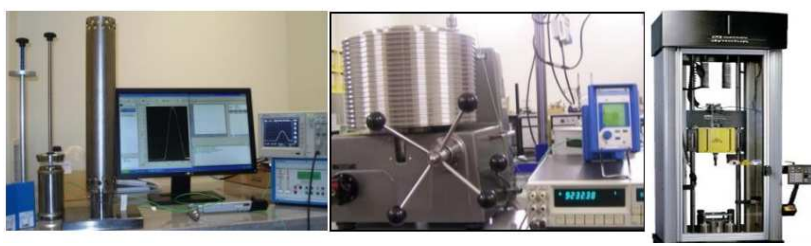

Fig. 1. Dynamic pressure systems; mass drop system, deadweight tester and impact test system, respectively.

of applied pressure and corresponding peak value of output signal of the transducer is described in standards mentioned above. However for dynamic calibration of the pressure transducer there is no international standard. Several NMIs and a few manufacturers have supplied different types of instruments and calibration systems as dynamic applications. Quick opening valves, negative pressure drop systems, shock tubes, drop weight etc. systems are some of the dynamic systems (Fig. 1) and they are used in the tests and calibrations of dynamic pressure transducers $[4,5]$. Impact test machines principally work as systems mentioned and the pulse achieved from these kinds of machines shall be used as a dynamic pressure source.

\section{Dynamic pressure measurements using impact test machine}

Impact test machines transfer the kinetic energy to tested material using termination probes which can be in different geometries. This energy is produced by free fallen or thrown straight down certain mass. Since the impact test machine uses the same principle like drop mass systems, there is a possibility of using obtained impacts in calibration of dynamic pressure sensors as dynamic pressure source [6]. Starting from this idea Dynatup 9250HV impact test machine was modified to make comparison calibration for dynamic pressure transducer, Fig. 1. In the modified system, the impact test machine and drop mass system was used together. The modified system provided us a computer controlled and repeatable dynamic data. For measurements two dynamic pressure transducers based on piezoelectricity were selected. Specifications of transducers are given in Table I. The transducer Kistler Model 6213BM was used as reference. Piston cylinder unit, shown in Fig. 2 schematically, is directly located under the vertical axis of probes which is connected to drop mass. Piston, test and reference dynamic pressure transducers are mounted on to a volume. This volume was filled by different types of oils. Sebecate

\section{TABLE I}

Specifications of Pressure Transducers Used in Measurements.

\begin{tabular}{c|c|c|c}
\hline \hline Manufacturer & Model & $\begin{array}{c}\text { Measurement } \\
\text { Range [MPa] }\end{array}$ & $\begin{array}{c}\text { Sensitivity } \\
{[\mathrm{pC} / \text { bar] }}\end{array}$ \\
\hline Kistler & $6229 \mathrm{AK}$ & $500 \mathrm{MPa}$ & 2.402 \\
Kistler & $6213 \mathrm{BK}$ & $800 \mathrm{MPa}$ & 1.193
\end{tabular}

and Drosera MS5 type oils were used in measurements as transmitting media. The transducers' outputs were connected to oscilloscope through conditioning amplifier and then voltage outputs of the measurement chain were transferred to PC in digital form. Typical measurement set up is given in Fig. 3.

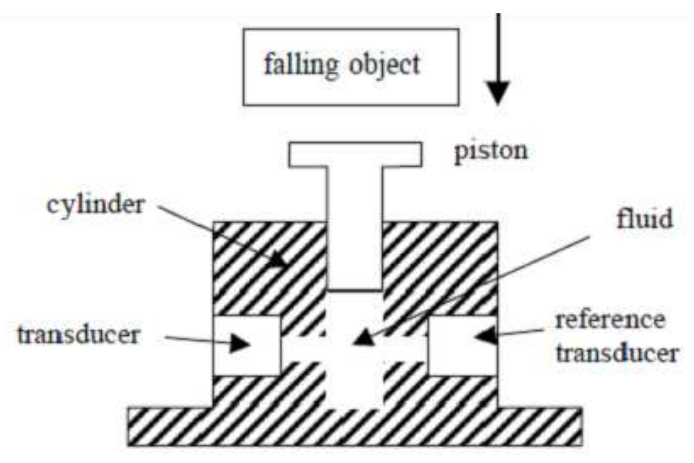

Fig. 2. Cylindrical pressure cell and pressure transducers.

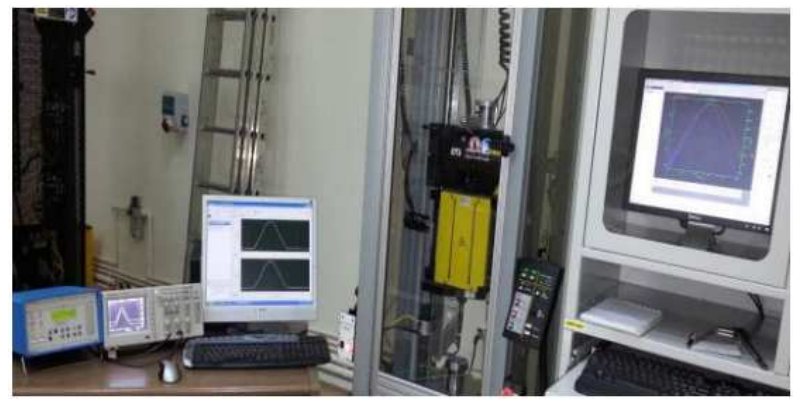

Fig. 3. Measurement setup used for comparison calibration of pressure transducers.

Measurements were performed at nominal pressure values of $100 \mathrm{MPa}, 150 \mathrm{MPa}, 200 \mathrm{MPa}, 250 \mathrm{MPa}, 300 \mathrm{MPa}$, $400 \mathrm{MPa}$ and $500 \mathrm{MPa}$. Typical waveforms of output signals of measurement chain for input pressures from $100 \mathrm{MPa}$ (yellow line) to $500 \mathrm{MPa}$ (blue line) are illustrated in Fig. 4. During collection of the data two different sampling rate and resolution used, $250 \mathrm{kS} / \mathrm{s}, 4$ bit and $800 \mathrm{kS} / \mathrm{s}, 12$ bit. It is clear that the results for the data collected with high sampling rate and resolution is more accurate. Because there is no internationally accepted method for determining peak values of the output signals, corresponding to dynamic pressure, different approximations were made such as fitting the data in the range of $90 \%$ of the maximum value of the output signals. Then the sensitivity of the pressure transducer being calibrated was calculated using Eq. (1). Models used for fitting the output signal "pulse" described below.

- Fit 1: Only peak amplitude of pulse is taken into account,

- Fit 2: Pulse signal fitted to parabola " $f(x)=a+$ $b x+c x^{2} "$ 


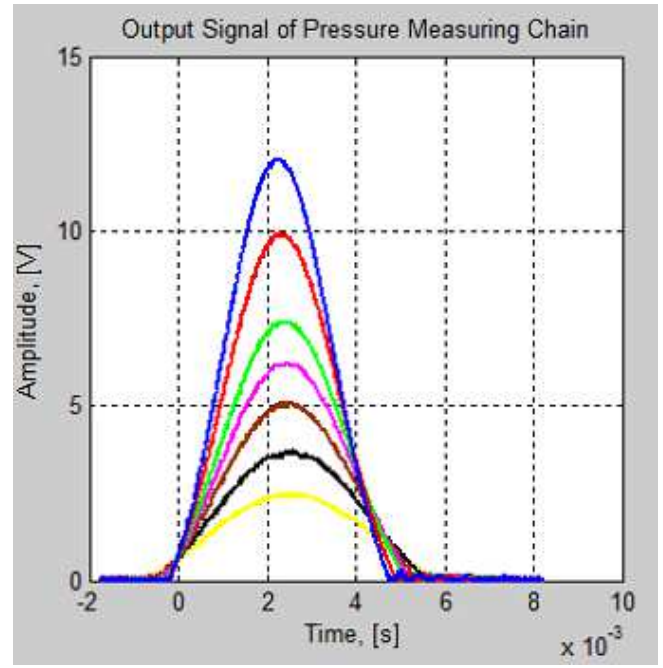

Fig. 4. Output signals of measuring chain for pressures $300 \mathrm{MPa}$ to $500 \mathrm{MPa}$.

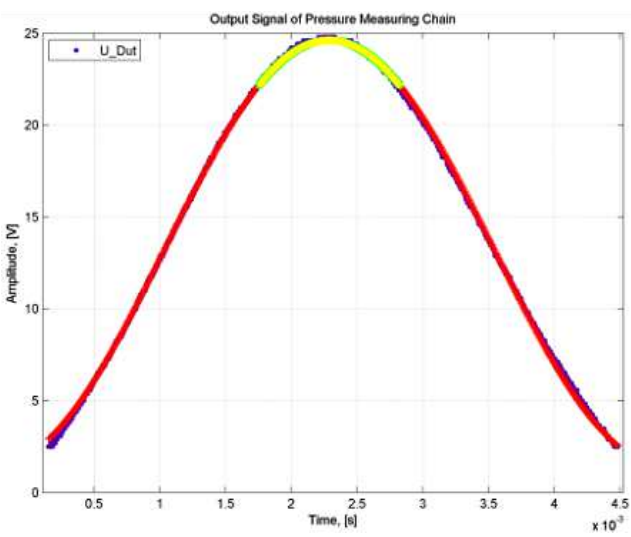

Fig. 5. Schematic presentation of the fitted pulse for the $90 \%$ of the maximum peak value.

- Fit 3: Pulse signal fitted to " $f(x)=A \sin (\omega x+\varphi)$ ", then fitted to parabola for the $90 \%$ of the maximum peak value, Fig. 5 ,

- Fit 4: Pulse signal fitted to " $f(x)=A_{1} \sin \left(\omega_{1} x+\right.$ $\left.\varphi_{1}\right)+A_{2} \sin \left(\omega_{2} x+\varphi_{2}\right)$ " then fitted to parabola for the $90 \%$ of the maximum peak value,

- Fit 5: Pulse signal fitted to " $f(x)=A_{1} \sin (\omega x)+$ $A_{2} \cos (\omega x)$ " then fitted to parabola for the $90 \%$ of the maximum peak value,

- Fit 6: Pulse signal fitted to " $f(x)=A_{1} \exp (-(x-$ $\left.\left.\left.B_{1}\right) / C_{1}\right)^{2}\right)+A_{2} \exp \left(-\left(x-B_{2}\right) / C_{2}\right)^{2}$ then fitted to parabola for the $90 \%$ of the maximum peak value.

$$
S_{\text {dut }}=S_{\text {ref }} \frac{u_{\text {dut,peak }}}{u_{\text {ref,peak }}},
$$

where, $S_{\text {dut }}$ is sensitivity of the calibrated transducer, $S_{\text {ref }}$ is sensitivity of the reference transducer, $u_{\text {dut,peak }}$ is maximum peak value of output of calibrated transducer and $u_{\text {ref,peak }}$ is maximum peak values of output of the reference transducer. Results are given in Fig. 6 and in Table II.

Dynamic pressure sensitivity values of the Kistler Model 6229AK transducer and associated conditioning amplifier were calculated for all fit types and for all types of fluid stated in Table II. Then, these sensitivities were normalized by dividing them to static pressure sensitivity of the pressure transducer. Then average and range of the relative error calculated for all fit types at discrete pressures were obtained.

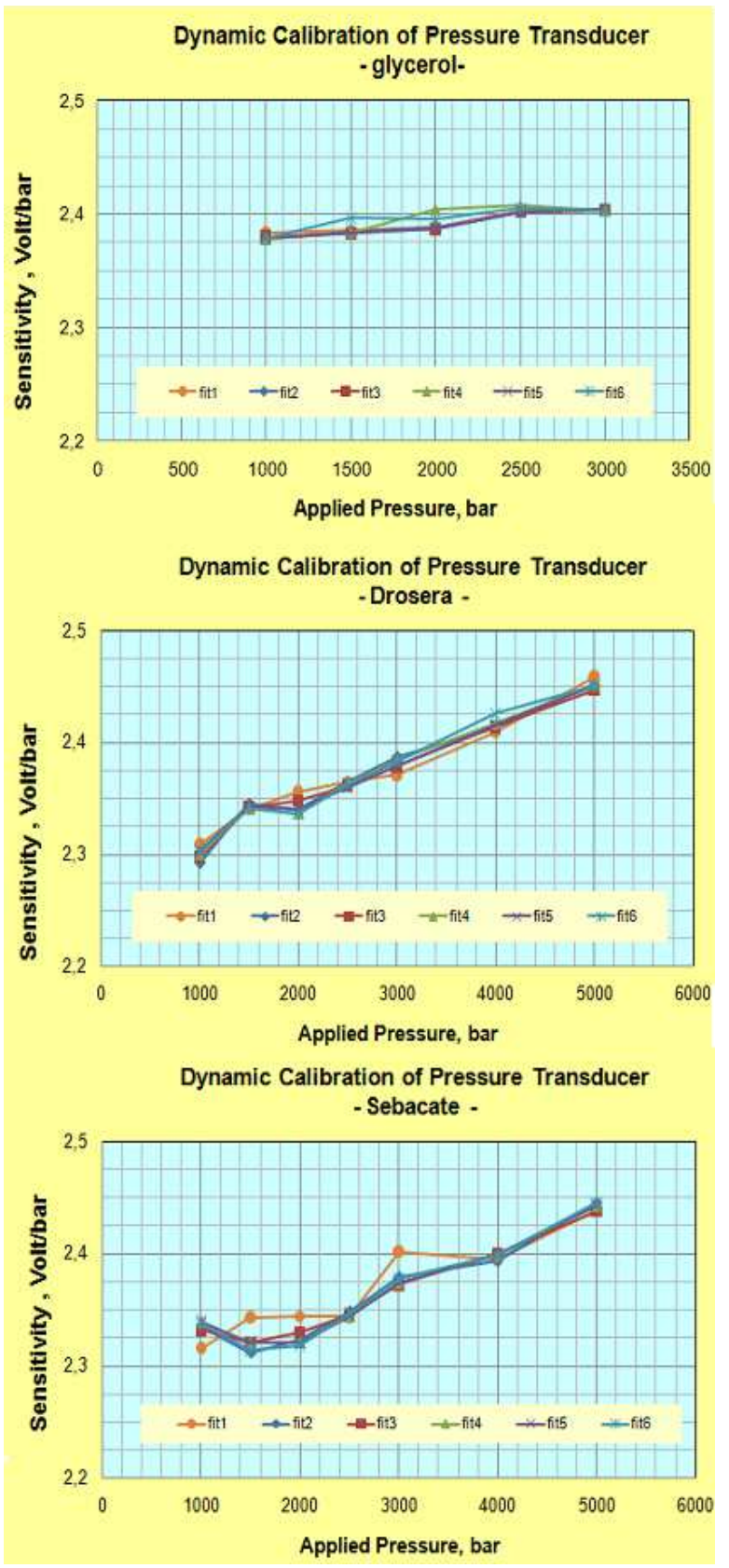

Fig. 6. Dynamic sensitivity values of measurement chain for fit 1 to fit 6 , sampling rate and resolution (a) $800 \mathrm{kS} / \mathrm{s}, 12 \mathrm{bit}$, (b) and (c) $250 \mathrm{kS} / \mathrm{s}, 4$ bit. 
TABLE II

Relative error on dynamic pressure sensitivities.

\begin{tabular}{c|c|c|c|c|c|c|c|c|c}
\hline \hline \multirow{2}{*}{$\begin{array}{c}\text { Pressure } \\
{[\mathrm{MPa}]}\end{array}$} & \multicolumn{9}{|c|}{ Gelative error, \% } \\
\cline { 2 - 10 } & \multicolumn{3}{|c|}{ Glycerol } & \multicolumn{3}{c}{ Drosera } & \multicolumn{3}{c}{ Sebacate } \\
\cline { 2 - 9 } & Mean & + Range & - Range & Mean & + Range & - Range & Mean & + Range & - Range \\
\hline 100 & -0.92 & 0.17 & -0.07 & -4.20 & 0.36 & -0.37 & -2.85 & 0.26 & -0.72 \\
150 & -0.66 & 0.43 & -0.13 & -2.48 & 0.12 & -0.08 & -3.33 & 0.89 & -0.43 \\
200 & -0.43 & 0.53 & -0.20 & -2.45 & 0.56 & -0.26 & -3.14 & 0.74 & -0.33 \\
250 & 0.06 & 0.17 & -0.08 & -1.62 & 0.10 & -0.14 & -2.34 & 0.13 & -0.08 \\
300 & 0.07 & 0.02 & -0.02 & -0.62 & 0.94 & -0.35 & -0.96 & 0.65 & -0.26 \\
400 & - & - & - & 0.58 & 0.42 & -0.26 & -0.19 & 0.09 & -0.14 \\
500 & - & - & - & 2.08 & 0.30 & -0.20 & 1.69 & 0.14 & -0.19
\end{tabular}

\section{Conclusion}

Dynamic pressure calibration of the pressure measuring chain was carried out. Range of the relative error for all fit types is found within 1\%. Relative error increases due to the pressure increase. This is probably the limitation of transducer's operating range. Bias occurs between result obtained for fluids Glycerol and Drosera-Sebacate. It is assumed that this is not only resulted from fluid but also the different sampling rate and resolutions of the measurement setups and also leakage and temperature effects. Authors prefer to use of Fit 3 for the calculation of the dynamic pressure sensitivity of the pressure transducer and/or measurement chain.

\section{References}

[1] International vocabulary of metrology - Basic and general concepts and associated terms (VIM), 3rd edition 2008 version with minor corrections, JCGM 2012.

[2] IND09 Dynamic: Traceable dynamic measurement of mechanical quantities, Annex Ia - JRP-Protocol, EMRP.

[3] V.E. Bean, Metrologia 30, 737 (1993/94).

[4] J. Hjelmgren, Dynamic Measurement of Pressure - A Literature Survey, SP Measurement Technology Report, SP Swedish National Testing and Research Institute, 2002, p. 34.

[5] Y. Durgut and I. Koçaş, in: VII. Ulusal Ölçüm Bilim Kongresi, İzmir 2008.

[6] Y.Durgut, E.Bağcl, S.Fank, B.Aydemir, in: 7th International Advanced Technologies Symposium (IATS'13), Istanbul 2013. 